hep-ph/9712454

FTUV/97-70

IFIC $/ 97-101$

FISIST/13-97/CFIF

\title{
Bounds on sterile neutrino mixing for cosmologically interesting mass range *
}

\author{
H. Nunokawa ${ }^{\mathrm{a}}$, J. T. Peltoniemi ${ }^{\mathrm{b}}$, A. Rossi ${ }^{\mathrm{c}}$ and J.W.F.Valle ${ }^{a}$ \\ ${ }^{a}$ Instituto de Física Corpuscular - C.S.I.C., Departament de Física Teòrica, Universitat de València \\ 46100 Burjassot, València, Spain \\ ${ }^{\mathrm{b}}$ Department of Physics, Box 9, 00014 University of Helsinki, Finland \\ ${ }^{\mathrm{c}}$ Dept. de Fisica, Inst. Superior Tecnico, 1096 Lisbon Codex, Portugal
}

\begin{abstract}
This talk summarizes our recent work which studied the impact of resonant $\nu_{e} \rightarrow \nu_{s}$ and $\bar{\nu}_{e} \rightarrow \bar{\nu}_{s}\left(\nu_{s}\right.$ is a sterile neutrino) conversions on supernova physics, under the assumption that the mass of the sterile state is in the few $\mathrm{eV}$-cosmologically significant range.
\end{abstract}

\section{Introduction}

It has been discussed that the resonant conversion (MSW effect) [1] of electron neutrinos into some sterile state in the dense media of type-II supernova could lead to some nontrivial consequences [2 [5]. We have reanalysed the impact of such conversion on supernova physics assuming the mass of the sterile state to be in the cosmologically significant range, i.e. $1-100 \mathrm{eV}$, the range relevant as dark matter component in the universe [6]

In what follows we will consider the system of $\nu_{e}$ and $\nu_{s}$ (and their anti-partners ) with nonzero masses and mixings and neglect the mixing between other flavors. The mass spectrum of neutrinos with the sterile state, relevant for our discussion, could naturally appear in some models 7].

\section{The active-sterile neutrino resonant conversion}

The effective potential for the $\nu_{e}-\nu_{s}$ system is given by,

$V_{e}=\frac{\sqrt{2} G_{F} \rho}{m_{N}}\left(Y_{e}-\frac{1}{2} Y_{n}\right)=\frac{\sqrt{2} G_{F}}{2 m_{N}} \rho\left(3 Y_{e}-1\right),(1)$

where $G_{F}$ is the Fermi constant, $\rho$ is the matter density, $m_{N}$ is the nucleon mass and $Y_{e}$ and

\footnotetext{
*Talk given by H. Nunokawa in Taup97, Gran Sasso, Italy,
} 7-11 September, 1997
$Y_{n}=1-Y_{e}$ are the net electron and neutron number per baryon, respectively. For anti-neutrino system $V_{e}$ should be replaced by $-V_{e}$.

The resonance condition is given as

$V_{e}=\frac{\delta m^{2}}{2 E_{\nu}} \cos 2 \theta$,

where $\delta m^{2}$ is the mass squared difference, $E_{\nu}$ is the neutrino energy and $\theta$ is the mixing angle. We assume $\delta m^{2}>0$, i.e., the heavier state is mostly sterile state.

In general, in the region above the neutrinosphere the density decreases as $r$ (the radial distance from the center) increases. On the other hand, the electron fraction $Y_{e}$ takes minimum value nearby the neutrinosphere due to the efficient neutronization process and then $Y_{e}$ increases as $r$ increases.

In Fig. 1 (a) the behavior of the matter density $\rho$ and the electron fraction $Y_{e}$ above the neutrinosphere are schematically shown. In Fig. 1 (b) we plot the behavior of the potential which can be inferred from the behaviors of $\rho$ and $Y_{e}$ in (a).

From Fig. 1 (b) we notice that for $\delta m^{2}>$ 0 as neutrinos leave from the neutrinosphere to the outer region, first $\bar{\nu}_{e}$ 's undergo resonant conversion and then $\nu_{e}$ 's are converted. The latter could undergo the transition twice if the values of $\left(\delta m^{2} / 2 E\right) \cos 2 \theta$ is smaller than the maximum value of $V_{e}$ (see Fig. 1(b)). In this work we appropriately take into account the double resonances for the $\nu_{e} \rightarrow \nu_{s}$ channel. 


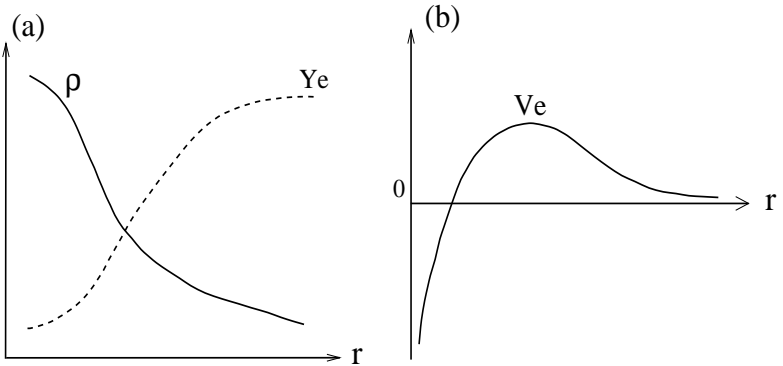

Figure 1. In (a) the behavior of the density $\rho$ and $Y_{e}$ above neutrinosphere are schematically plotted and in (b) the potential $V_{e} \propto \rho\left(3 Y_{e}-1\right)$ is plotted.

\section{Impact of the conversion on supernova physics}

In this section we discuss the effect of $\nu_{e} \rightarrow \nu_{s}$ and $\bar{\nu}_{e} \rightarrow \bar{\nu}_{s}$ conversions on shock re-heating, $\bar{\nu}_{e}$ signal and heavy elements nucleosynthesis using the $\rho$ and $Y_{e}$ profiles from Wilson's supernova model.

\subsection{Shock re-heating}

We first consider the neutrino-conversion effect on shock re-heating in the delayed explosion scenario [8]. We estimate the neutrino energy deposition rate at the stalled shock with and without conversion and take the ratio which is defined to be $R$. It is clear that disappearance of either $\nu_{e}$ or $\bar{\nu}_{e}$ due to the resonant conversion into the sterile states will reduce the rate $R$. In Fig. 2 we plot the iso-contour for different values of the ratio $R$ in the parameter space $\left(\delta m^{2}, \sin ^{2} 2 \theta\right)$. We can conclude that if the neutrino re-heating is essential for successful supernova explosion the parameter region right to the curve, say $R=0.5$, is disfavoured.

\subsection{SN1987A $\bar{\nu}_{e}$ signal}

Next we consider the impact on the observation of $\bar{\nu}_{e}$ signal on the earth. It is also clear that the resonant conversion of $\bar{\nu}_{e} \rightarrow \bar{\nu}_{s}$ could induce a reduction of $\bar{\nu}_{e}$ signal in the terrestrial detector. In Fig. 3 we present the contours of the $\bar{\nu}_{e}$ survival probability, properly averaged over the neutrino energy. We conclude that the successful observation of the $\bar{\nu}_{e}$ signal from supernova SN1987A in

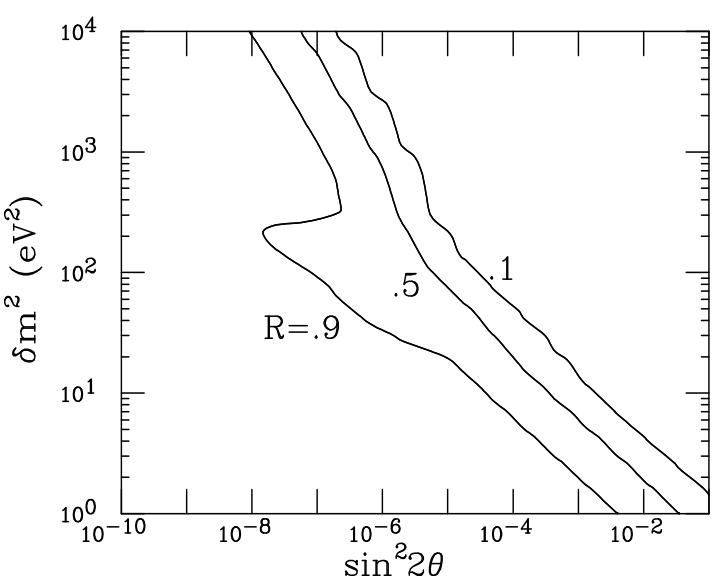

Figure 2. Contour plot of the ratio $R$ of the neutrino energy deposition behind the shock wave in the presence of conversions into sterile neutrinos, versus the case without conversions.

Kamiokande and IMB detectors 10 implies the absence of significant conversion of $\bar{\nu}_{e} \rightarrow \bar{\nu}_{s}$, disfavouring the parameter region right to the curve, say $P=0.5$.

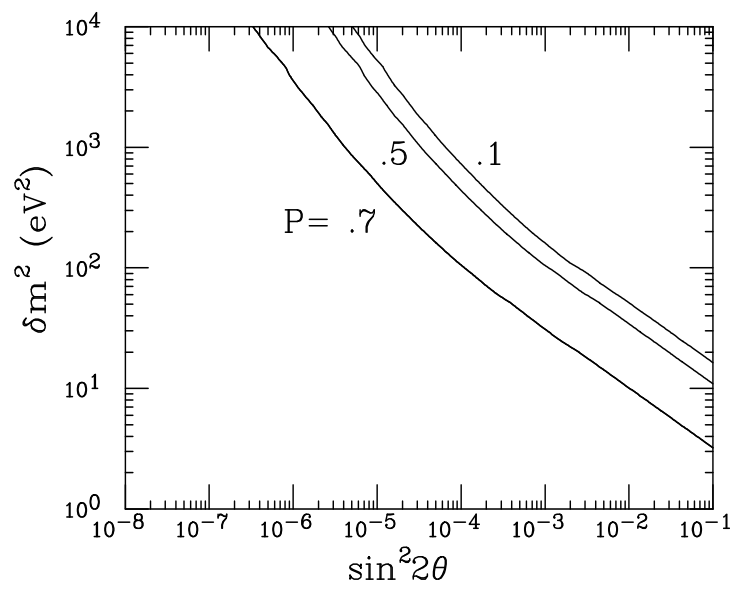

Figure 3. Contour plots of the survival probability $P$ (figures at the curve) for the $\bar{\nu}_{e} \rightarrow \bar{\nu}_{s}$ conversion.

\section{3. r-process}

Finally we discuss the impact of the neutrino conversion on heavy elements nucleosynthesis, so called $r$-process in supernova [9]. As discussed in ref. [9] one of the most relevant physical parame- 
ter in the $r$-process is the electron fraction $Y_{e}$. To have successful $r$-process the site must be neutron rich, i.e. $Y_{e}<0.5$. The $Y_{e}$ value is mainly determined by the competition between the following two absorption reactions:

$\nu_{e}+n \rightarrow p+e^{-}$,

$\bar{\nu}_{e}+p \rightarrow n+e^{+}$.

In the standard supernova model the latter process is favoured due to the higher average energy of $\bar{\nu}_{e}$ guaranteeing the neutron richness.

We expect that $\nu_{e} \rightarrow \nu_{s}\left(\bar{\nu}_{e} \rightarrow \bar{\nu}_{s}\right)$ conversion induce a decrease (increase) of $Y_{e}$ due to the decrease of the first (second) neutrino-absorption reaction in eq. (3). The decrease of $Y_{e}$ implies that the site becomes more neutron rich and the $r$-process could be enhanced [5] whereas the increase of $Y_{e}$ induces the suppression of the $r$ process. Therefore, depending on which conversion channel is more efficient the $r$-process could either be enhanced or suppressed. In Fig. 4 we present the effect of neutrino conversion on the value of $Y_{e}$. In the region right to the curve $Y_{e}=$ 0.5 the value of $Y_{e}$ is larger than 0.5 and hence the $r$-process is suppressed. On the other hand, in the region delimited by the curve $Y_{e}=0.4$ the value of $Y_{e}$ could be decreased compared to the standard case, leading to the enhancement of $r$-process. We note that due to non-trivial "feedback" effect the value of $Y_{e}$ is not expected to be smaller than $1 / 3$. See ref. [11] for more discussion.

\section{Conclusion}

We have studied the impact of the resonant conversion of electron neutrinos into sterile state whose mass is assumed to be in the cosmologically interesting range. We have derived bounds on neutrino parameters from the shock re-heating, SN1987A $\bar{\nu}_{e}$ signal as well as $r$-process and we also found some parameter region where $r$-process could be enhanced. More detailed discussion on this work is found in ref. 11.

\section{REFERENCES}

1. S. P. Mikheyev and A. Yu. Smirnov, Sov. J. Nucl. Phys. 42 (1986) 913; Sov. Phys. Usp. 30

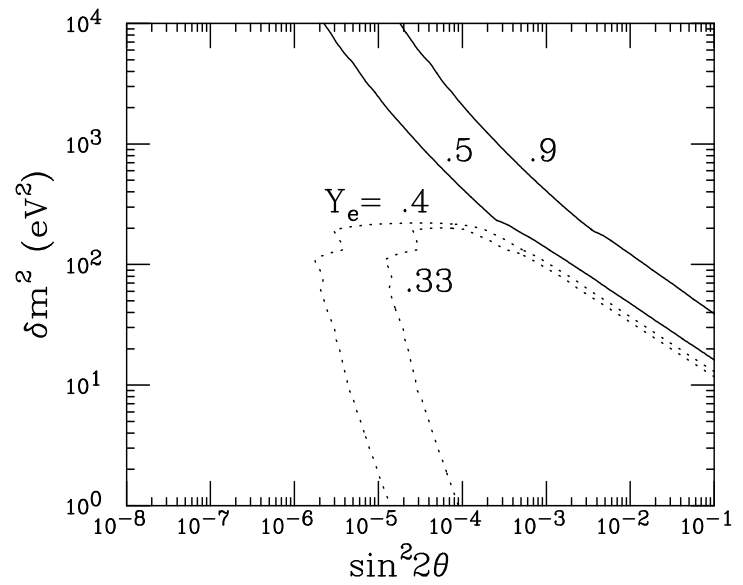

Figure 4. Contour plots for the electron concentration $Y_{e}$ (figures at the curves) in the region relevant for $r$-process.

(1987) 759; L. Wolfenstein, Phys. Rev D17 (1978) 2369; ibid D20 (1979) 2634.

2. S. P. Mikheyev and A. Yu. Smirnov, Sov. Phys, JETP, 64 (1986) 4; Prog. Part. Nucl. Phys. 23 (1989) 41.

3. G. Raffelt and G. Sigl, Astropart. Phys. 1 (1993) 165.

4. X. Shi and G. Sigl, Phys. Lett. B323 (1994) 360 .

5. J. T. Peltoniemi, Proc. Third Tallinn Syposium on Neutrino Physics, Ed. I. Ots, J. Lõhmus, P. Helde and L. Palgi (Tartu, 1995) p. 103; hep-ph/9511323; hep-ph/9506228.

6. See for e.g., E. W. Kolb and M. S. Turner, The Early Universe (Addison-Wesley, 1990).

7. J. T. Peltoniemi, D. Tommasini and J. W. F. Valle, Phys. Lett. B298 (1993) 383.

8. J. R. Wilson, Numerical Astrophysics ed. J. M. Centrella, J. M. Leblanc and R. L. Bowers (Boston, Jones and Bartlett), p.422 (1983).

9. Y.-Z. Qian et al., Phys. Rev. Lett.71 (1993) 1965.

10. K. Hirata et. al., Phys. Rev. Lett. 58 (1987) 1490; R. Bionta et. al., Phys. Rev. Lett. 58 (1987) 1494.

11. H. Nunokawa, J. T. Peltoniemi, A. Rossi and J.W.F.Valle, Phys. Rev. D56 (1997) 1704. 